\title{
Underwater polypectomy: successful endoscopic resection of a large gastric pedunculated polyp
}

\author{
Keiichi Kimura, Shunsuke Yoshii, Yoshito Hayashi, Tetsuo Takehara \\ Osaka University Graduate School of Medicine, Japan
}

Polypectomy is the standard therapy for gastrointestinal pedunculated polyps [1]. However, achieving effective countertraction to snare the stalk of the polyp located in the direction of gravity can sometimes be difficult [2]. Herein, we present a case of a large gastric polyp located on the greater curvature of the gastric body that was resected using the "water immersion" technique in order to overcome the technical difficulty.

A 75-year-old woman was referred to our hospital because of anemia. A large hyperplastic polyp with oozing blood was diagnosed by esophagogastroduodenoscopy. The polyp lay on the greater curvature of the gastric body and it was difficult to maintain adequate visibility of the polyp stalk. Therefore, we used the "water immersion" technique in order to ensure adequate visualization of the polyp morphology. The stomach was filled with water via the water-jet function of the single channel endoscope (GIF-Q260J, Olympus Medical Systems Corp., Tokyo, Japan). The floating of the polyp head made the polyp "stand up" in the water and the root of the stalk was well visualized. Polypectomy was performed using a standard polypectomy snare (Captivator II, Boston Scientific, Marlborough, MA, USA) and an electrosurgical generator (VIO300D, ERBE, Tübingen, Germany). Complete resection was successfully achieved with no adverse event (Fig. 1). Histological examination of the resected specimen revealed a gastric hyperplastic polyp (Fig. 2).

Water immersion can improve visibility and traction for resection [3]. Underwater polypectomy can be a useful method for pedunculated polyps located in the direction of gravity.

Department of Gastroenterology and Hepatology, Osaka University Graduate School of Medicine, Japan

\section{Conflict of Interest: None}

Correspondence to: Tetsuo Takehara, MD, PhD, Department of Gastroenterology and Hepatology, Osaka University Graduate School of Medicine, 2-2 Yamadaoka, Suita, Osaka, Japan, e-mail: takehara@gh.med.osaka-u.ac.jp

Received 25 May 2017; accepted 30 May 2017 published online 26 July 2017

DOI: https://doi.org/10.20524/aog.2017.0182
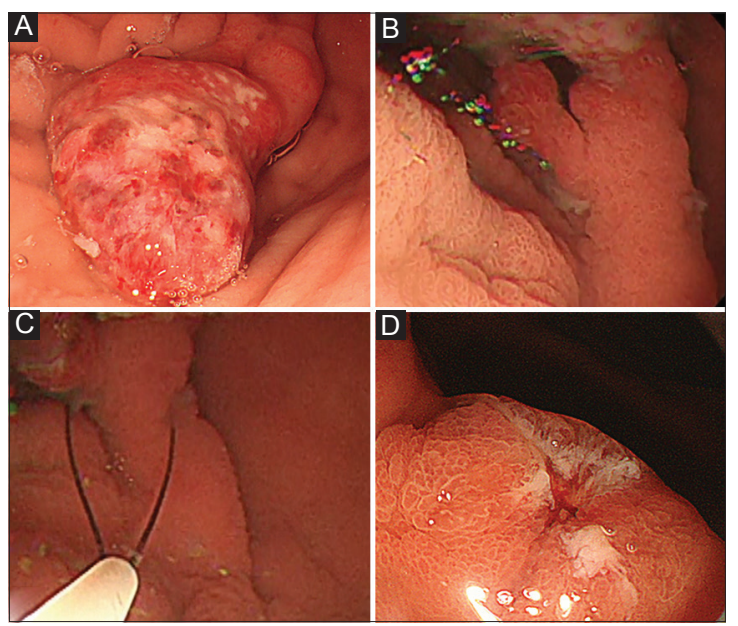

Figure 1 Underwater view during the procedure. (A) Conventional endoscopic view of a large pedunculated polyp on the greater curvature of the gastric body. (B) The stalk of the polyp was well visualized. (C) Underwater polypectomy was performed using a standard polypectomy snare. (D) The ulcer bed after underwater polypectomy
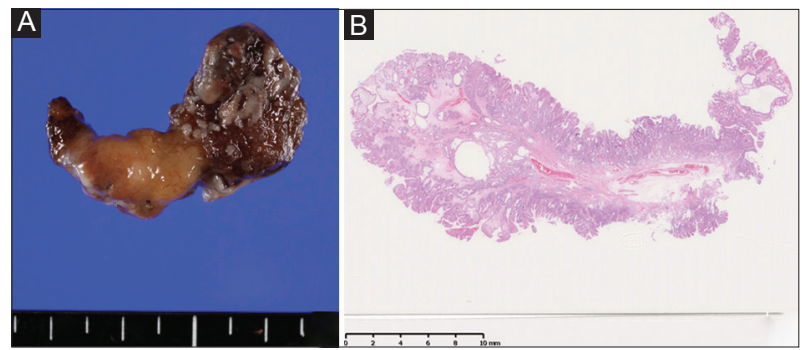

Figure 2 Histological images following underwater polypectomy. (A) The resected specimen. (B) Histological examination of the polyp specimen revealed elongated, branched, and dilated gastric foveolae, with areas of chronic and active inflammation

\section{References}

1. Tursi A, Brandimarte G. Endoscopic polypectomy of large pedunculated gastric polyps using a new, safe, and effective technique. Endoscopy 2002;34:673-674.

2. Noda H, Ogasawara N, Koshino A, et al. Thread-traction with a sheath of polypectomy snare facilitates endoscopic submucosal dissection of early gastric cancers. Gastroenterol Res Pract 2016;2016:9415497.

3. Yoshii S, Hayashi Y, Matsui T, et al. "Underwater" endoscopic submucosal dissection: a novel technique for complete resection of a rectal neuroendocrine tumor. Endoscopy 2016;48 Suppl 1 UCTN:E67-E68. 